\title{
ANEXO DEL MANEJO DEL CARCINOMA MAMARIO POR ESTADOS CLINICOS
}

\section{ESTADOS TEMPRANOS}

\section{Carcinoma In Situ (Estado 0-Tis No M0)}

Consultar capítulo de Carcinoma In Situ

\section{Carcinoma Infiltrante}

TX Tumor Primario de tamaño no conocido que ha sido manipulado y no se conocen sus dimensiones.

Conducta: se debe resecar el área quirúrgica incluyendo la cicatriz, con margen suficiente.

Si no hay temor residual se clasifica como TO NO y la conducta complementaria debe ser discutida de acuerdo con cada caso en particular.

Si hay tumor residual microscópico con márgenes libres se debe realizar disección axilar de nivel 1 y 2 .

Si hay tumor residual con márgenes positivos debe practicarse Mastectomía Radical Modificada.

Todas las pacientes sometidas a cirugía conservadora deben recibir RADIOTERAPIA en la mama y QUIMIOTERAPIA ADYUVANTE.

Las pacientes sometidas a MRM deben recibir quimioterapia Adyuvante.

Las pacientes premenopáusicas con RE $(+)$ y todas las post-menopáusicas, independientemente del estado de los receptores deben recibir hormonoterapia.

Estado I (T1 N0 M0)

1. Cirugía conservadora con vaciamiento axilar y radioterapia complementaria.

2. Mastectomía radical modificada si no cumple requisitos para cirugía conservadora

3. Quimioterapia co-adyuvante según junta multidisciplinaria. Por compromiso ganglionar axilar o sin compromiso ganglionar axilar con factores pronósticos adversos (edad, receptores hormonales negativos, tamaño tumoral, estudio genético, estudios complementarios).

Estado II A (TO y Tl con N1, T2 N0) y Estado II B (T2 N1 y T3 N0)

1. Cirugía conservadora si cumple requisitos, con vaciamiento completo más radioterapia

2. Si la relación seno-tumor no permite cirugía conservadora se puede ofrecer quimioterapia de inducción, como en estados localmente avanzados, con la intención de salvar este impedimento según acuerdo por junta multidisciplinaria.

3. Quimioterapia co-adyuvante en paciente con indicaciones de la misma.

Pacientes con compromiso ganglionar axilar.

Pacientes con tumores mayores de $2 \mathrm{cms}$.

Pacientes sin compromiso ganglionar pero con factores pronóstico adversos.

\author{
Esquemas de Primera Línea \\ AC: Adriamicina $60 \mathrm{mg} / \mathrm{m} 2 \mathrm{IV}$ ( día 1 ) \\ Ciclofosfamida $600 \mathrm{mg} / \mathrm{m} 2$ IV ( día 1 ) \\ Profilaxis antiemética agresiva y obligatoria \\ 4 ciclos, 1 cada 3 semanas, para pacientes \\ premenopaúsicas. \\ Iniciar 2-4 semanas después de la cirugía \\ CMF: Ciclofosfamida $600 \mathrm{mg} / \mathrm{m} 2$ IV (día 1 y 8 ) \\ Methotrexate $40 \mathrm{mg} / \mathrm{m} 2$ IV (día 1 y 8 ) \\ 5 fluoruracilo $600 \mathrm{mg} / \mathrm{m} 2$ IV (día 1 y 8 ) \\ Profilaxis antiemética agresiva y obligatoria \\ 6 ciclos, 1 cada 4 semanas, para pacientes \\ postmenopaúsicas. \\ Iniciar 2-4 semanas después de la cirugía
}

4. Mastectomía radical modificada en pacientes que no pueden recibir radioterapia (embarazadas o con enfermedad del tejido colágeno).

5. Pacientes con mastectomía radical modificada y ganglios negativos no requieren radioterapia.

6. Mastectomía radical modificada en pacientes con tumores mayores de $3 \mathrm{cms}$. (no aprobados para quimioterapia primaria por la junta multidisciplinaria) más radioterapia (ver anexo), más quimioterapia según esquemas y/ o hormonoterapia, según las siguientes indicaciones:

. Pacientes con compromiso ganglionar axilar.

Pacientes con tumores mayores de $2 \mathrm{cms}$.

. Pacientes sin compromiso ganglionar pero con factores pronósticos adversos.

Pacientes post- menopáusicas con receptores hormonales positivos como tratamiento co-adyuvante.

Todas las pacientes serán sometidas a tratamiento hormonal complementario después del tratamiento multidisciplinario (cirugía, radioterapia, quimioterapia) de acuerdo a estudio funcional gonadal.

\section{Esquemas de Hormonoterapia}

\section{Antiestrógenos}

Tamoxifén 20 mgrs al día

Toremifeno 60 mgrs al día

\section{Progestinas}

Acetato de Megestrol 160 mgrs al día

Inhibidores de Aromatasa

Anastrazol $1 \mathrm{mgr}$ al día

Aminoglutetimida 150-500 mgrs 
Agonistas de LHRH

Goserelín 3,5 mgrs cada 4 semanas

Leuprolide 3,75 mgrs cada 4 semanas

Andrógenos

Testosterona 250 mgrs cada 2 semanas

\section{Estrógenos}

7. Quimioterapia neoadyuvante, en los tumores adheridos a la fascia pectoral mayor. Durante el acto quirúrgico siempre se debe incluír la resección del músculo subyacente al tumor para obtener mayor posibilidad de márgenes libres y por lo tanto menor riesgo de recurrencia local.

Pacientes con estado funcional comprometidos según la escala de Karnofsky salen de protocolo y recibirán manejo por cuidados paliativos.

\section{ANEXO DE TRATAMIENTO CON RADIOTERAPIA PARA ESTADOS TEMPRANOS}

Tratamiento Conservador del Carcinoma Mamario con Axila Negativa:

1. Carcinoma In Situ.

2. Carcinoma Infiltrante estados I - IIA - IIB

Volumen a irradiar: la totalidad del seno.

Dosis tiempo y fraccionamiento: puede ser 180 - 200 o $250 \mathrm{cGy}, 5$ sesiones por semana hasta dosis total que oscila entre 4.000 y $5.000 \mathrm{cGy}$.

Sobreimpresión: únicamente se da a los Carcinomas Infiltrantes.

Dosis tiempo y fraccionamiento: 180 - 200 o 250 cGy, 5 sesiones por semana hasta 1.400 a $2.000 \mathrm{cGy}$

\section{Tratamiento Conservador del Carcinoma Mamario} con Axila Positiva:

Estado IIB con axila positiva:

Volumen a irradiar: se irradia el seno, la axila y la fosa supraclavicular.

Dosis tiempo y fraccionamiento: Puede ser 180 - 200 o $250 \mathrm{cGy}, 5$ sesiones por semana hasta una dosis total de 4.000 a $5.000 \mathrm{cGy}$.

Si la paciente conserva la mama recibirá sobreimpresión que se hará en igual forma que para los estados iniciales.

\section{ESTADOS LOCALMENTE AVANZADOS}

Estado IIIA (T0, T1, o T2 con N2 y T3 con N1 o N2) Estado IIIB ( $T 4$ con cualquier $N$ o cualquier $T$ con N3)

1. Las pacientes con estados localmente avanzados deben recibir los 3 tipos de terapia.

2. Las pacientes con estados localmente avanzados no deben ir inicialmente a cirugía.

3. Las pacientes con estados localmente avanzados deben recibir quimioterapia primaria (neoadyuvante o de inducción) 3 ciclos según estos esquemas:

\section{Esquema de Primera Línea}

AC: Adriamicina $60 \mathrm{mg} / \mathrm{m} 2$ IV (Día 1) Ciclofosfamida $600 \mathrm{mg} / \mathrm{m} 2$ IV (Día 1)

Profilaxis Antiemesis Ondansetrón (infusión) $24 \mathrm{mg} .30 \mathrm{~min}$. antes.

4 Ciclos, uno cada 3 semanas para pacientes premenopáusicas

Iniciar 2-4 semanas después de la cirugía

CMF: Ciclofosfamida $600 \mathrm{mg} / \mathrm{m} 2$ IV (día 1 y 8)

Methotrexate $40 \mathrm{mg} / \mathrm{m} 2$ IV (día 1 y 8)

5 fluoruracilo $600 \mathrm{mg} / \mathrm{m} 2$ IV (día 1 y 8 )

Profilaxis antiemesis obligatoria con ondansetrón

4 Ciclos, uno cada 4 semanas para pacientes post menopáusicas

Iniciar 2-4 semanas después de la cirugía

Todas las pacientes deben ser evaluadas previa aplicación de cada ciclo. En caso de no respuesta o progresión tumoral después de 2 ó máximo 3 ciclos de tratamiento, será valorada la conveniencia de radioterapia previa al tratamiento quirúrgico.

4. Mastectomía radical modificada: en caso de respuesta clínica a la quimioterapia primaria, siempre y cuando no existan criterios de inoperabilidad.

5. Quimioterapia adyuvante: para pacientes con enfermedad localmente avanzada después de tratamiento con quimioterapia neoadyuvante y cirugía o pacientes con enfermedad temprana tratada mediante cirugía y con indicación de quimioterapia.

Esquema de primera línea: el esquema a utilizar depende de la respuesta clínica obtenida durante la quimioterapia primaria y el resultado de la patología quirúrgica. En caso de haber logrado respuesta clínica parcial, mayor del $75 \%$, o respuesta clínica completa se continuará con el mismo esquema hasta completar 6 ciclos. En caso contrario se debe cambiar el esquema según decisión de junta.

- Esquema AC. Protocolo arriba anotado

- Esquema CMF. Protocolo arriba anotado

- Vinorelbine $30 \mathrm{mg} / \mathrm{m} 2$ IV (días 1 y 8) Esteroide 30 min. antes aplicar esta droga a través de catéter venoso central con irrigación venosa posterior a la aplicación del antineoplásico.

.5 Fluoruracilo $600 \mathrm{mg} / \mathrm{m} 2$ íV (días 1 y 8 )

Esquemas de segunda línea 0 rescate:

MMM Mutamicín $7 \mathrm{mg} / \mathrm{m} 2$ IV (día 1) cada 6 semanas

Methotrexate $30 \mathrm{mg} / \mathrm{m} 2$ IV (día 1 y 8 )

Mitoxantrone $7 \mathrm{mg} / \mathrm{m} 2$ IV cada 3 semanas 


\section{TAXOL $175 \mathrm{mg} / \mathrm{m} 2$ IV en infusión de 3 horas a través de filtro \\ Indispensable para este tipo de medica- mento premedicar con:}

\author{
. Dexametasona $16 \mathrm{mg} .6$ y 12 horas pre- \\ vios a la aplicación de taxol \\ . Clemastina $2 \mathrm{mg}$ IV $30 \mathrm{~min}$. previos a la \\ aplicación del taxol \\ . Ranitidina $50 \mathrm{mg}$ IV $30 \mathrm{~min}$. previos a la \\ apliación del taxol
}

\section{Ciclos cada 3 semanas}

6. La hormonoterapia: estará indicada en pacientes post menopáusicas con receptores hormonales positivos, en cuyo caso reemplazará a la quimioterapia adyuvante.

7. Radioterapia: luego de quimioterapia neoadyuvante sin respuesta favorable. Si hay respuesta a la radioterapia, se considerará el tratamiento quirúrgico.

8. Radioterapia: en todas las pacientes tratadas con quimioterapia neoadyuvante más cirugía más quimioterapia adyuvante, sin importar el compromiso tumoral residual ni el compromiso tumoral ganglionar axilar.

La radioterapia deberá iniciarse a más tardar 6 semanas después de haber concluído el tratamiento con quimioterapia adyuvante o 4 semanas después de haber sido considerada como progresión tumoral o sin respuesta a la quimioterapia neoadyuvante.

Después de la cirugía independientemente del resultado de patología la paciente recibirá quimioterapia y luego radioterapia complementaria, posteriormente deberá recibir tratamiento hormonal.

El tratamiento hormonal suplementario después de la quimioterapia adyuvante estará indicado en todas las pacientes post menopáusicas. Además las pacientes premenopáusicas con receptores hormonales positivos, una vez sometidas al estudio de la función gonadal (con rangos para menopausia) también deberán recibir tratamiento hormonal.

\section{ANEXO DE TRATAMIENTO CON RADIOTERAPIA PARA ESTADOS LOCALMENTE AVANZADOS IIIA- IIIB}

1. Radioterapia preoperatoria en casos de no respuesta a la quimioterapia.

Volúmen a irradiar: totalidad de la mama, axila y fosa supraclavicular.

Dosis tiempo y fraccionamiento: puede ser 180 - 200 o 250 cGy 5 sesiones por semana hasta unadosis que oscila entre 4.000 y $5.000 \mathrm{cGy}$.

La sobredosis se hace de acuerdo a la respuesta clínica.

2. Radioterapia post operatoria.

Volúmen a irradiar: pared torácica, axila y fosa supraclavicualr.

Dosis tiempo y fraccionamiento: puede ser $180-200$ o 250 cGy 5 sesiones por semana hasta una dosis que oscila entre 4.000 y $5.000 \mathrm{cGy}$.

\section{CARCINOMA INFLAMATORIO (T4d)}

Carcinoma raro, muy agresivo, de evolución rápida (menor de 6 meses); en general se diagnostica clínicamente. Se presenta como un proceso inflamatorio con eritema y edema en más de la tercera parte de la mama y la patología demuestra compromiso tumoral de los linfáticos subdérmicos; puede presentar en algunos casos masa dominante pero usualmente no se puede definir por el compromiso difuso. A la palpación se pueden encontrar nódulos satélites dentro del parénquima. La mamografía muestra engrosamiento de la piel con un proceso infiltrativo. La mayoría son pobremente diferenciados; son de muy mal pronóstico.

El manejo es igual al estado IIIB:

Quimioterapia con base en antraciclinas independiente del estado menopáusico.

Radioterapia a la totalidad de la mama y drenajes ganglionares.

Dosis, tiempo y fraccionamiento 250 cGy en 5 sesiones por semana hasta una dosis de 4.000 a 4.500 cGy.

Cirugía solo estaría indicada para pacientes libres de enfermedad luego del tratamiento inicial con Quimio y Radioterapia.

Sobredosis si no es posible efectuar cirugía y de acuerdo con la respuesta clínica.

En algunos casos seleccionados y de acuerdo a criterio médico se irradiará la cadena mamaria homolateral.

\section{ESTADO IV}

Los estados IV no son quirúrgicos. La cirugía tiene únicamente carácter higiénico - paliativo.

Toda paciente con estado IV comprobado debe recibir el siguiente tratamiento:

Quimioterapia: con intención paliativa esquema CMF, como esquema de primera línea.

Radioterapia: los pacientes con metástasis en fosa supraclavicular y/o con edema de la piel por fuera de la mama y que se logre respuesta a la quimioterapia. Se dará radioterapia como en los estados localmente avanzados.

Las metástasis serán tratadas en forma paliativa individualmente para cada paciente.

Las pacientes con estado IV por fosa supraclavicular o por edema de la piel con respuesta clínica adecuada a la quimioterapia primaria serán tratadas como un estado IIIB.

Las pacientes premenopáusicas con evidencia de función ovárica comprobada con mediciones de FSH y Estradiol luego de 3 ciclos de quimioterapia deben ser sometidas a ooforectomía.

Hormonoterapia en forma secuencial posterior a la quimioterapia y que durará hasta que exista evidencia de progresión tumoral.

La radioteapia en el estado IV se usa para manejo paliativo según decisión de junta multidisciplinaria.

\section{HORMONOTERAPIA}

La hormonoterapia está indicada en las siguientes situaciones: 
1. Paciente post menopáusica con receptores hormonales positivos, cuando exista indicación de tratamiento sistémico como alternativa a la quimioterapia.

2. En pacientes post-menopáusicas después de haber sido sometidas a quimioterapia independiente de los receptores hormonales.

3. En paciente premenopáusica con receptores hormonales positivos posterior a la quimioterapia.

4. En el estado IV administrado en forma secuencial teniendo en cuenta que se debe esperar un mínimo de 3 meses para obtener respuesta antes de cambiar de terapia. Se utiliza el siguiente esquema:

\section{Hormonoterapia}

\section{Paciente Premenopáusica:}

1. Ooforectomía quirúrgica o actínica, si después de la Quimioterapia existe evidencia de actividad ovárica.

2. Terapia hormonal, de acuerdo con esquema. Se inicia tratamiento con antiestrógenos. Si no hay respuesta podrá realizarse ooforectomía en mayores de 35 años o utilizar análogos de LH RH.

\section{Paciente Postmenopáusica:}

\section{Terapia hormonal}

Se inicia con antiestrógenos y si no hay respuesta y no se considera la quimioterapia se puede dar anastrasol o acetato de magestrol.

En caso de progresión de la enfermedad durante la hormonoterapia, podría utilizarse un segundo agente hormonal si se considera justificado por no existir otra alternativa terapéutica, o en caso positivo podrían utili- zarse esquemas de Quimioterapia de 2da. y 3ra. línea, o con protocolos de intensificación de dosis.

La mayor experiencia hasta ahora ha sido con el Tamoxifeno en dosis de 20 mgrs al día por 5 años; las pacientes sometidas a este tratamiento deben tener vigilancia ginecológica por el riesgo bajo para cáncer endometrial cuando tienen útero. El Raloxifeno parece tener menos efectos estrogénicos secundarios.

El Acetato de Megestrol y el Anastrazole tienen respuestas muy similares y se pueden utilizar como esquemas de segunda línea hormonal. Este medicamento está recomendado solo en la postmenopausia. La aminoglutetimida ha caído en desuso por sus efectos secundarios tan marcados.

Los análogos de GNRH pueden utilizarse como segunda o tercera línea de hormonoterapia en la premenopáusica. Porcentaje de respuestas del 32 al $42 \%$.

Los andrógenos se usan poco por sus efectos virilizantes.

\section{CANCER MAMARIO EN USA. ESTADOS CLINICOS Y} RESULTADOS DE TRATAMIENTO 1995

\begin{tabular}{llllll}
\hline Estado & 0 & I & II & III & IV \\
Presentación & $12.4 \%$ & $41.8 \%$ & $33.1 \%$ & $8.0 \%$ & $4.7 \%$ \\
Tratamiento & $\mathrm{C}(\mathrm{R})$ & $\mathrm{C}(\mathrm{Q}, \mathrm{R})$ & $\mathrm{C}, \mathrm{Q}(\mathrm{R})$ & $\mathrm{Q}, \mathrm{C}, \mathrm{R}$ & $\mathrm{Q}, \mathrm{R}$ \\
Fin & Curat. & Curat. & Curat. & Curat. & Paliativo \\
Sobrevida & $98 \%$ & $90 \%$ & $70 \%$ & $50 \%$ & $15 \%$ \\
\hline
\end{tabular}

$\mathrm{C}=$ cirugía

$\mathrm{Q}=$ quimioterapia

$(\mathrm{R})=$ radioterapia

\section{LECTURAS CONSULTADAS Y RECOMENDADAS}

1. Deerases of the Breast, Harris et al. Lippincott-Raven Publishers, Philadelphia 1996.

2. The Breast, Bland/Copeland, III W.B. Sanders Co. Philadelphia 1991.

3. Gynecologic Oncology. William Hoskins, Carlos A. Pérez, Robert C, Young. Lippincott-Raven Publishers. Philadelphia 1996.

4. Gynecologic Cancer Surgery, Paul Morrow. Churchill Livingstone Inc. 1996.

5. Practical Gynecologic Oncology. Berek-Hacker. Williams and Wilkins. Baltimore, Maryland, USA. 1995.

6. Medical Oncology. A Comprehensive Review. University of Texas. MD Anderson Cancer Center. Richard Pazdur, MD. EPR. Huntingtom. N. York. 1992.
7. Atlas de Cirugía de la Mama. Samuel A. Wells, Junior, V. Leroy Young, Dorothy A. Andriole. Mosby Doyma Libros Madrid 1995.

8. Atlas de Ecografia Mamaria comparada, PB Guyer K.C. Dewbury. Ediciones Doyma. Barcelona 1993.

9. Cancer de la Mama, Oscar Acebedo Ferrer, Editora.

10. Clinical Gynecologic Oncology, Philip J Dissia, Wilham T, Creasman, Mosby INC. St Louis Missouri, USA 1997.

11. Gynecologic Oncology. Malcom Coppleson. Churchill Livingston, London, U.K. 1992.

12. Te Linde. Ginécología Quirúrgica. 7a. Edición. John D. Thompson, John A. Roch. Editorial Médica Panamericana, Buenos Aires, 1992.

13. Cirugía Oncológica. Umberto Veronesi. Unions Tipografico Editrice Torinese. Torino, Italia 1991. 